\title{
Reconfigurable hyperbolic metamaterial with negative refraction
}

\author{
Harish N. S. Krishnamoorthy ${ }^{1}$, Behrad Gholipour ${ }^{2}$, Nikolay I. Zheludev ${ }^{1,2}$ and Cesare Soci ${ }^{1}$ \\ ${ }^{I}$ Center for Disruptive Photonic Technologies, Nanyang Technological University, Singapore \\ ${ }^{2}$ Optoelectronics Research Centre \& Centre for Photonic Metamaterials, University of Southampton, UK \\ Correspondence: harish.k@ntu.edu.sg
}

\begin{abstract}
Relying on the phase change property of GST chalcogenide glass, we demonstrate a novel reconfigurable hyperbolic metamaterial in which the spectral region of negative refraction can be switched from near infra-red to visible.

OCIS codes: (160.3918) Metamaterials; (250.5403) Plasmonics.
\end{abstract}

Hyperbolic metamaterials (HMMs) are metal-dielectric nano-composites with the unique ability to support large wave-vector electromagnetic states, which have been exploited in a wide range of applications such as imaging [1,2] and quantum optics [3-5]. The onset frequency of hyperbolic dispersion and its character (Type-I or Type-II) are determined by the constituent metal and dielectric materials and their fill-fractions, and usually cannot be changed once the HMM is fabricated. Recently, phase change materials such as vanadium dioxide or chalcogenide glasses have emerged as a unique platform enabling nanophotonic switches and optically reconfigurable metasurfaces $[6,7]$, opening up the possibility to develop tunable HMMs [8]. Here we use the chalcogenide alloy $\mathrm{Ge}_{2} \mathrm{Sb}_{2} \mathrm{Te}_{5}$ (GST), which has traditionally been exploited as the switchable medium in rewritable optical disks and non-volatile electronic memories, as one of the constituents of a multilayer HMM. By exploiting the reversible amorphous to crystalline phase change property of GST, we demonstrate a Type-I HMM in which the spectral region of negative refraction can be switched from the near infra-red (IR) to visible upon thermal annealing.

The HMM comprises of 7 periods of alternating layers of silver and GST of thicknesses $15 \mathrm{~nm}$ and $24 \mathrm{~nm}$, respectively, as shown schematically in the inset of Figure 1(A). An ultrathin layer ( $\sim \mathrm{nm}$ thick) of germanium is used as a wetting layer in order to ensure good quality silver films. The HMM is deposited on pre-cleaned glass microscope slides by RF sputtering in high-purity argon gas.
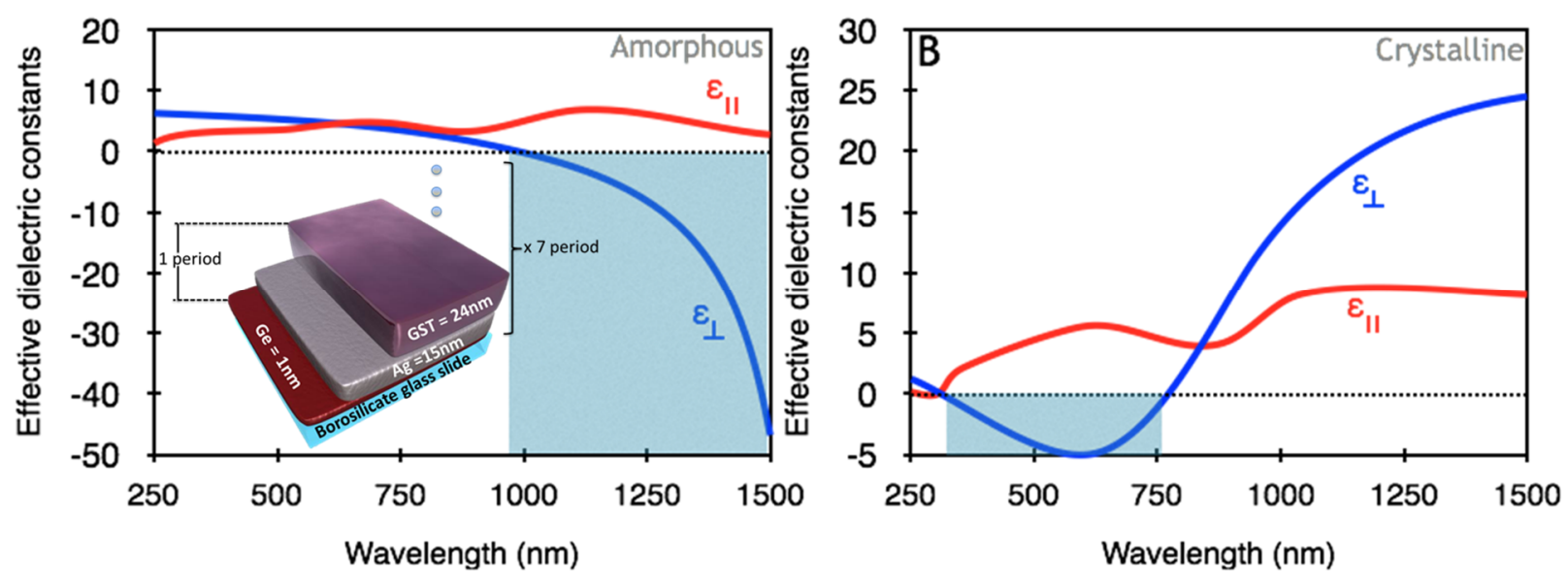

Fig. 1. Effective dielectric constants of the HMM extracted from ellipsometry with GST layers in the (A) amorphous and (B) crystalline phases, respectively. The shaded part indicates the region of hyperbolic dispersion. Schematic of the structure is shown in the inset of Fig. 1(A).

Optical characterization of the HMM was performed by carrying out ellipsometric measurements of the HMM sample. The dielectric tensor of such a layered nano-composite takes the form, $\overleftrightarrow{\varepsilon}(\vec{r})=\operatorname{diag}\left(\varepsilon_{x x}, \varepsilon_{y y}, \varepsilon_{z z}\right)$, where $\varepsilon_{x x}=\varepsilon_{y y}=\varepsilon_{\|}$and $\varepsilon_{z z}=\varepsilon_{\perp}$. By using a uniaxial anisotropic model, the effective in-plane $\left(\varepsilon_{\|}\right)$and out-of-plane $\left(\varepsilon_{\perp}\right)$ dielectric constants of the HMM were determined from ellipsometry $[9,10]$. When the GST layers in the HMM are in the amorphous phase, the HMM shows Type-I hyperbolic dispersion $\left(\varepsilon_{\perp}<0, \varepsilon_{\|}>0\right)$ in the near-IR region and elliptical dispersion $\left(\varepsilon_{\perp}, \varepsilon_{\|}>0\right)$ in the visible region, as shown in Figure 1(A). Subsequently, the sample is annealed above the GST crystallisation temperature $\left(\sim 160^{\circ} \mathrm{C}\right)$, to convert the GST phase from amorphous to crystalline. 
Effective dielectric constants of the annealed nanocomposite extracted from ellipsometry measurements are shown in Figure 1(B); with GST layers in the crystalline phase, the HMM exhibits Type-I hyperbolic dispersion in the visible region and elliptical dispersion in the near-IR region.
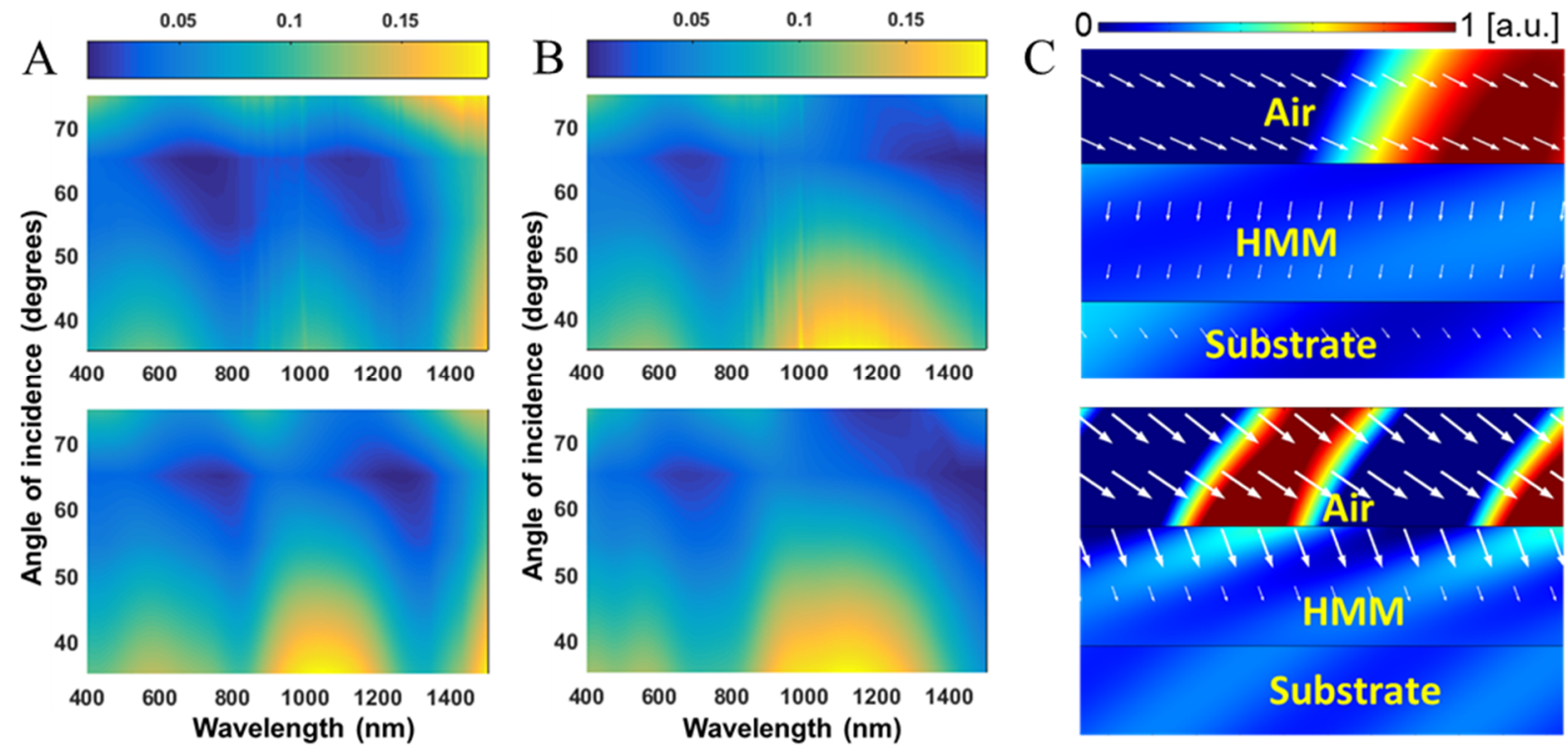

Fig. 2. Experimental (top panel) and calculated (bottom panel) reflection spectra as a function of angle for TM polarized light incident on the HMM, with GST layers in the (A) amorphous, and (B) crystalline phases. (C) Color map of the electric field and the direction of power flow for TM polarized plane wave incident on the amorphous HMM at 60 degree angle, at wavelengths $1200 \mathrm{~nm}$ (top panel) and $600 \mathrm{~nm}$ (bottom panel).

Polarized reflection measurements as a function of incidence angle were carried out on the HMM sample in both the amorphous and crystalline phases to verify the accuracy of the dielectric constants extracted from ellipsometry measurements. Figures 2(A) and 2(B) show the good agreement between the measured and calculated reflection spectra for TM polarization, for both as-deposited and annealed HMM samples.

Since the samples show Type-I hyperbolic dispersion, they are expected to show negative refraction as well. Figure 2(C) shows the electric field map and the Poynting vector for the HMM in the amorphous phase simulated using a finite element method. In the simulation, a plane wave with TM polarization is incident on the air-HMM interface at an angle of $60^{\circ}$. If the wavelength of the incident wave is $1200 \mathrm{~nm}$ (top panel), it experiences hyperbolic dispersion and undergoes negative refraction. On the other hand, if the incident light has a wavelength of $600 \mathrm{~nm}$ (bottom panel), it does not experience the strong anisotropy and is positively refracted. This situation is inverted after conversion of the GST layers to their crystalline phase, where similar simulations show negative refraction at $600 \mathrm{~nm}$ and positive refraction at $1200 \mathrm{~nm}$.

In conclusion, we demonstrated a hyperbolic metamaterial containing a chalcogenide phase change constituent in which both positive and negative refraction coexist in the visible and near-IR spectral regions, respectively; the sign of refraction in both spectral regions can be inverted by simply switching the phase of the chalcogenide glass.

[1] Z. Jacob, L. V. Alekseyev, and E. Narimanov, "Optical Hyperlens: Far-field imaging beyond the diffraction limit", Opt. Express 14, 8247 (2006).

[2] Z. Liu, H. Lee, Y. Xiong, C. Sun and X. Zhang, "Far-Field Optical Hyperlens Magnifying Sub-Diffraction-Limited Objects", Science 315, 1686 (2007).

[3] M. A. Noginov et al., "Controlling spontaneous emission with metamaterials", Opt. Lett. 35, 1863 (2010).

[4] Z. Jacob et al., "Engineering photonic density of states using metamaterials", Appl. Phys. B. 100, 215 (2010).

[5] H.N.S. Krishnamoorthy, Z. Jacob, E. Narimanov, I. Kretzschmar, and V.M. Menon, "Topological Transitions in Metamaterials", Science 336, 205 (2012).

[6] B. Gholipour, J. Zhang, K. F. MacDonald, D. W. Hewak, and N. I. Zheludev, Adv. Mater 25, 3050 (2013).

[7] Q. Wang, E. T. F. Rogers, B. Gholipour, C.-M. Wang, G. Yuan, J. Teng, and N. I. Zheludev, Nature Photonics 10, 60 (2015).

[8] H.N.S. Krishnamoorthy, Y. Zhou, S. Ramanathan, E. Narimanov, and V. M. Menon, "Tunable hyperbolic metamaterials using phase change heterostructures", Appl. Phys. Lett. 104, 121101 (2014).

[9] Gururaj V. Naik, Jingjing Liu, Alexander V. Kildishev, Vladimir M. Shalaev, and Alexandra Boltasseva, "Demonstration of Al:ZnO as a plasmonic component for near-infrared metamaterials", Proc. Natl. Acad. Sci. U.S.A. 23, 8834 (2012).

[10] T. Tumkur, Y. Barnakov, S. T. Kee, M. A. Noginov, and V. Liberman, "Permittivity evaluation of multilayered hyperbolic metamaterials: Ellipsometry vs.reflectometry", J. Appl. Phys. 117, 103104 (2015). 